\title{
Folding of an intrinsically disordered iron-binding peptide in response to sedimentation revealed by cryo-EM - Supporting Information
}

Geula Davidov ${ }^{\#, 1,2}$, Gili Abelya ${ }^{\#, 1}$, Ran Zalk $^{2}$, Benjamin Izbicki ${ }^{1}$, Sharon Shaibi ${ }^{1}$, Lior Spektor ${ }^{3}$, Dayana Shagidov ${ }^{3}$, Esther G. Meyron-Holtz ${ }^{3}$, Raz Zarivach $^{1,2,4}$, and Gabriel A. Frank ${ }^{*}, 1,2$

${ }^{1}$ Department of Life Sciences, Ben-Gurion University of the Negev, Beer Sheva, 8410501, Israel

${ }^{2}$ The National Institute for Biotechnology in the Negev, Ben-Gurion University of the Negev, Beer Sheva, 8410501, Israel

${ }^{3}$ Faculty of Biotechnology and Food Engineering, Technion-Israel Institute of Technology, Technion City, 32000, Haifa, Israel

${ }^{4}$ Ilse Katz Institute for Nanoscale Science and Technology, Ben-Gurion University of the Negev, Beer Sheva, 8410501, Israel

\footnotetext{
\# Contributed equally

* Corresponding Author
} 


\section{Ferritin-M6A self assembles and forms nano-cages in cells}

Size exclusion chromatography analysis of L-Ferritin-M6A

L-ferritin-M6A assembles and purifies from E. coli as nano-cages as apparent from its sizeexclusion chromatogram (Figure S1). Chromatography was performed as described in the methods section of the main text.

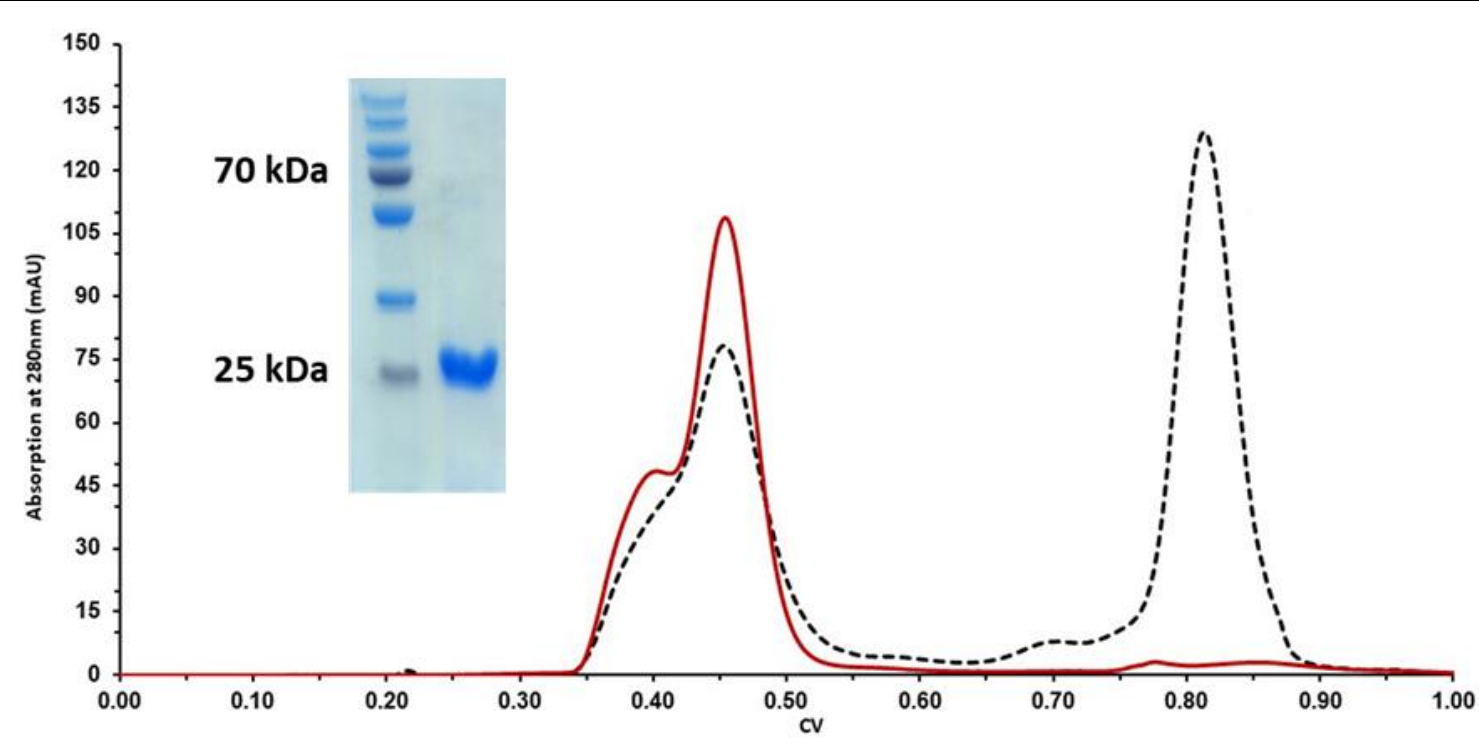

Figure S1: Size exclusion chromatograms of L-ferritin-M6A (Red), and of a calibration sample composed of a mixture of Thyroglobulin (669 kDa) and Ribonuclease A (13.7 kDa) (Black dashed line). The elution volumes of L-ferritin-M6A and of Thyroglobulin are similar, as expected from an assembled nano-cage. Chromatography was performed as described in the methods section. Protein for cryo-EM was collected from the center of the main peak. The inset shows an SDS-PAGE analysis of the isolated sample.

Consequently, the sediments found in the Untreated samples were formed in-vivo.

\section{Iron-loading of Ferritin-M6A in Mammalian cells}

We investigated the ability of murine L-ferritin-M6A to assemble to a 24-mer and incorporate iron in a human cell-line. The experiments were performed in HeLa cells with targeted deletion of the human $\mathrm{H}$-ferritin and L-ferritin subunits $\left(\mathrm{HeLa}^{\mathrm{FtKO}}\right)$, a kind gift of Dr. Maura Poli, from the 
University of Brescia, as described in [1]. $\mathrm{HeLa}^{\mathrm{FHO}}$ cells were transfected with plasmids coding for murine H-ferritin, L-ferritin, H-ferritin-M6A, L-ferritin-M6A, or combinations as indicated. Eighteen hours after the transfection, cells were incubated for 24 hours with $100 \mu \mathrm{g} / \mathrm{ml}$ ferric ammonium citrate (FAC). Cell lysates were prepared with $1 \%$ Triton lysis buffer, and $60 \mu \mathrm{g}$ protein/lane or $200 \mu \mathrm{g}$ protein/lane were loaded on gels for immunoblot and iron detection, respectively. Lysates were separated on an $8 \%$ poly-acrylamide native gel and evaluated by immunoblot-blot with anti-murine L-ferritin antibody (a kind gift from Prof. A.M. Konijn from the Hebrew University of Jerusalem). L-ferritin-M6A migrated at the same height as purified mouse liver ferritin, indicating that the L-ferritin-M6A assembled into a 24-mer in the $\mathrm{HeLa}^{\mathrm{FtKO}}$ cells (data not shown). With iron staining (DAB enhanced Perl's stain, (as described in [2]), we were unable to detect any iron in the L-ferritin-M6A homo-oligomer, in contrast to the positive control of murine H-ferritin-M6A (Figure S2a). A similar, but more sensitive method was carried out by loading ferritin with $1 \mu \mathrm{M} \mathrm{Fe}^{55}$ in the form of $\mathrm{FeCl}_{3}$. Following 24 hours of iron loading, iron was detected again in the murine H-ferritin, but not in the L-ferritin-M6A (Figure S2c, left side). We concluded that in the L-ferritin-M6A homo-oligomer iron accumulation is below the detection limit in the HeLa cell setting. However, we hypothesized that the M6A peptide might improve the efficiency of iron incorporation in an H- and L-M6A-ferritin hetero-oligomer, as it improved the iron accumulation in the murine H-ferritin-M6A homo-oligomer, compared to the wild type $\mathrm{H}$-ferritin homo-oligomer [3]. To verify that $\mathrm{H}$-ferritin and L-ferritin subunits assemble into hetero-oligomers, following the transfection into $\mathrm{HeLa}^{\mathrm{FtKO}}$ cells, we immunoprecipitated ferritin from metabolically labeled $\mathrm{HeLa}^{\mathrm{FtKO}}$ cells, that were transfected with either $\mathrm{H}$ - or with $\mathrm{L}$-, or with a combination of both $\mathrm{H}$ - and L-subunits, with antibodies specific for either H- or Lsubunits (Fig. S2b). These results indicated that hetero-oligomers are formed in the transfected 
$\mathrm{HeLa}^{\mathrm{FtKO}}$ cells. Based on this result, we conducted an experiment that included co-transfection of murine H-ferritin with either L-ferritin or L-ferritin-M6A at a ratio of 1:10 and 1:20, respectively. Following 24 hours of incubation with $1 \mu \mathrm{M} \mathrm{Fe}^{55}$, cells were lysed and proteins were separated on reducing SDS-PAGE (30 $\mu \mathrm{g}$ protein/lane) or on a native gel (70 $\mu \mathrm{g}$ protein/lane) for immunoblot and iron detection respectively (Figure S2c). The results supported that L-ferritinM6A undergoes oligomerization into 24-mer hetero- and homo-oligomer ferritin nano-cages and suggested that H-ferritin-L-ferritin-M6A hetero-oligomers may accumulate slightly more iron than the hetero-oligomer that contains H-ferritin-L-ferritin-WT, similarly to the observation reported in [3].

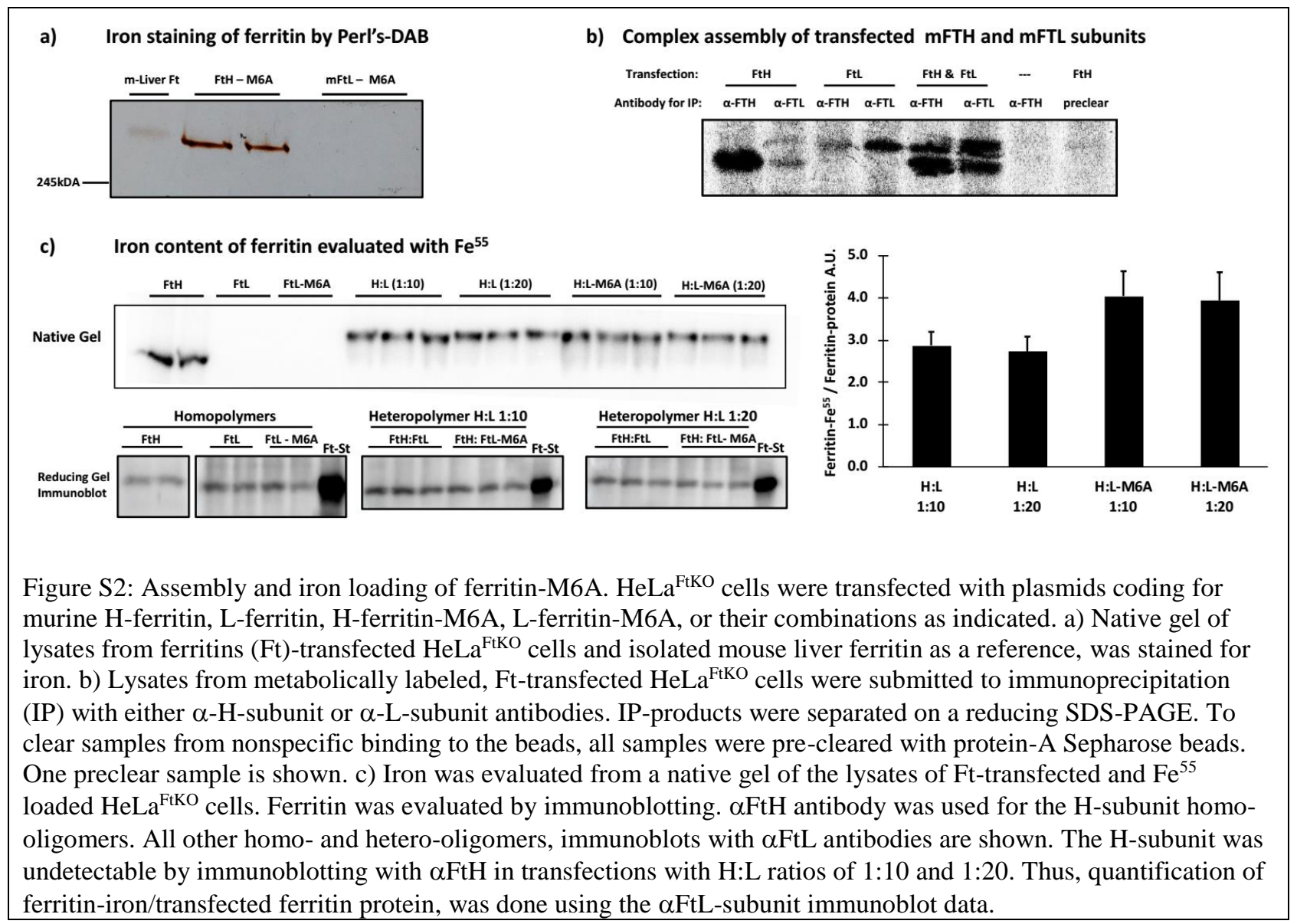




\section{$X$-ray crystallography data collection and structure determination}

\section{Data collection}

The L-ferritin-M6A-H crystal was cryo-protected. X-ray diffractions were collected on beamline ID23-1 at the European Synchrotron Radiation Facility (ESRF, Grenoble, France). The crystal diffracted to $1.7 \AA$ resolution. Dataset was measured at a wavelength of $0.977 \AA$ for a total of 3600 frames with an oscillation range of $0.1^{\circ}$ and an exposure time of $0.037 \mathrm{~s}$ per image. The crystal-to-detector distance was $240.64 \mathrm{~mm}$.

Structure determination, refinement and analysis:

Data reduction and scaling were performed by XDS. The output map and protein sequence were calculated via molecular replacement with mouse L-ferritin (PDB 1LB3). The final model was built by Coot and refined with REFMAC [4,5]. Structural figures and structure analysis were prepared with Chimera [6]. Data collection and refinement results are summarized in Table S1 and figure $\mathrm{S} 3$.

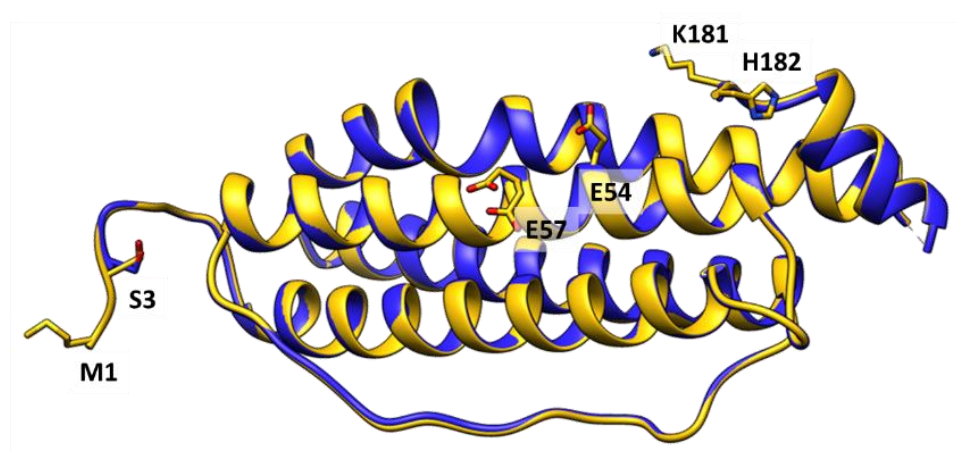

Figure S3: 1.7 Å resolution X-ray crystallography structure of Acetate Washed L-ferritin-M6A. Acetate Washed L-ferritin-M6A was crystallized and its structure was determined. The structure of mouse apo L-ferritin (PDB 1LBL, blue) and the structure of L-ferritin-M6A from this study (PDB 6Z3D, yellow) were almost identical (C $\alpha$ $\mathrm{RMSD}=25.5 \AA$ A calculated with 166 pairs of atoms). While the $\mathrm{N}$ and $\mathrm{C}$ terminal residues of L-ferritin (designated) were resolved in the new structure, M6A was unstructured. 


\begin{tabular}{|c|c|}
\hline Protein name & L-ferritin-M6A-H \\
\hline PDB code & $6 \mathrm{Z3D}$ \\
\hline Data collection & ESRF - ID23-1 \\
\hline Space group & P 42,2 \\
\hline \multicolumn{2}{|l|}{ Cell dimensions } \\
\hline $\mathrm{a}, \mathrm{b}, \mathrm{c}(\AA)$ & $134.30,134.30,166.85$ \\
\hline$\alpha, \beta, \gamma\left({ }^{\circ}\right)$ & $90,90,90$ \\
\hline Resolution $(\AA)$ & $1.7-166.85(1.70-1.74)$ \\
\hline Rmerge & $0.19(3.38)$ \\
\hline $\mathrm{I} / \sigma \mathrm{I}$ & $14.2(1.4)$ \\
\hline $\mathrm{CC}_{1 / 2}$ & $0.979(0.606)$ \\
\hline Completeness (\%) & $100(100)$ \\
\hline Redundancy & $26.5(26.5)$ \\
\hline Wavelength $(\AA)$ & 0.978 \\
\hline No. unique reflections & 166767 \\
\hline \multicolumn{2}{|l|}{ Refinement } \\
\hline Resolution $(\AA)$ & 1.70 \\
\hline Rwork/Rfree & $15.51 / 17.59$ \\
\hline \multicolumn{2}{|l|}{ No. atoms } \\
\hline Protein & A-1409, B-1412, C-1411, D-1416, E-1427, F-1407 \\
\hline Ligand/ion & $\mathrm{H}-24$ \\
\hline Water & G-1137 \\
\hline \multicolumn{2}{|l|}{$B$-factors } \\
\hline Protein & 26.27 \\
\hline Ligand/ion & 53.78 \\
\hline Water & 38.48 \\
\hline \multicolumn{2}{|l|}{$R M S D$} \\
\hline Bond lengths $(\AA)$ & 0.013 \\
\hline Bond angles $\left({ }^{\circ}\right)$ & 1.67 \\
\hline Ramachandran statistics * & P: 929 (97.69\%), A: 1 (2.21\%), O: 0 (0.11\%) \\
\hline Missing residues & $\begin{array}{l}\text { A: -19-1, 183-196, B:-19-0, 183-196, C:-19-0, 183-196, } \\
\text { D:-19-0, 183-196, E:-19-0, 183-196, F:-19-1, 184-196 }\end{array}$ \\
\hline
\end{tabular}




\section{Cryo-EM image processing}

Typical cryo-EM data collected and analyzed in this study (Figures S4-6 and table S2). Data was collected and processed as described in the main text.

(a)

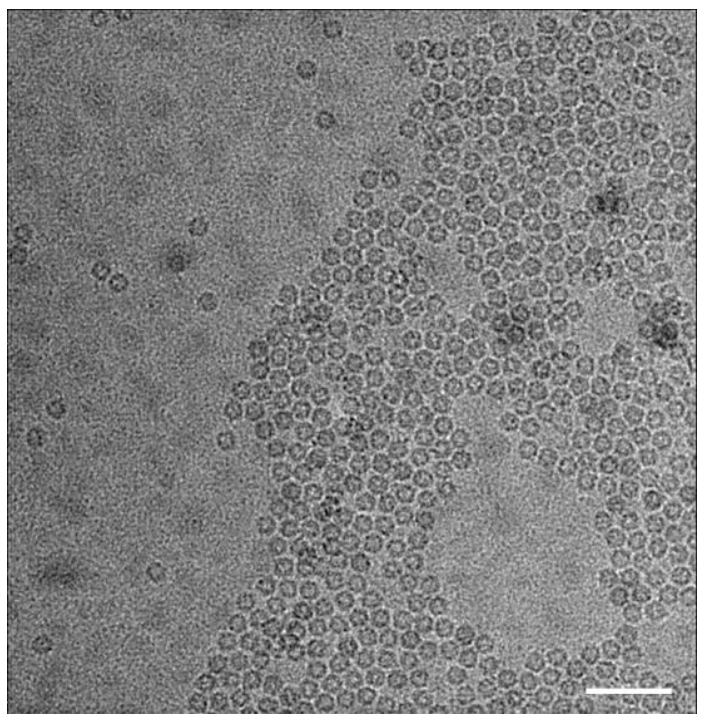

(c)

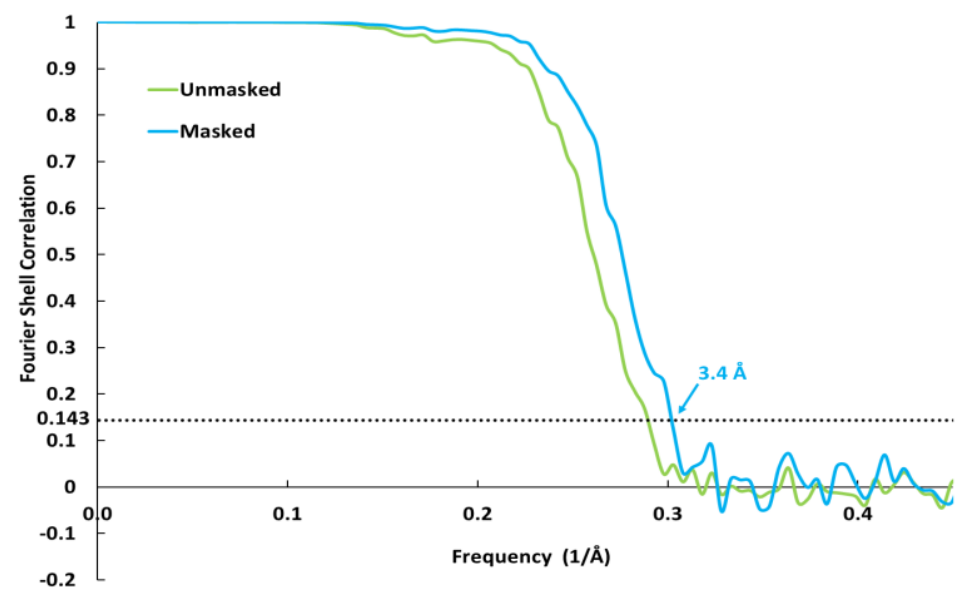

(b)

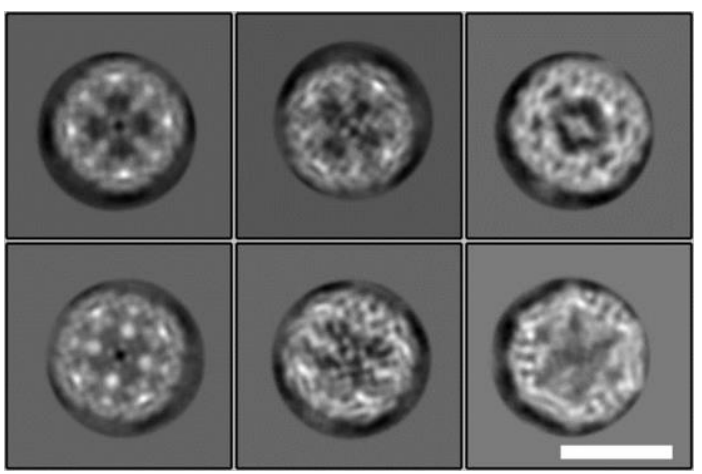

(d)
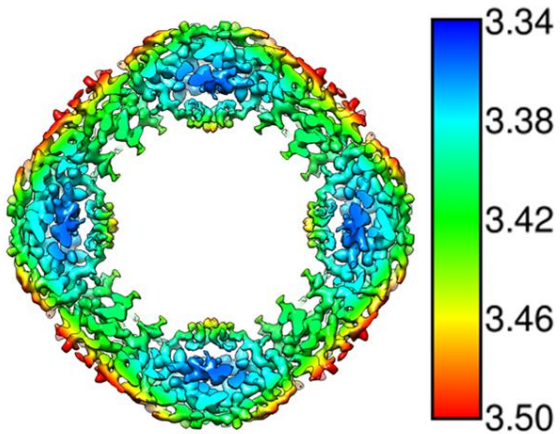

Figure S4: Summary of image processing results of the Untreated sample. A representative micrograph (a), typical "good" class averages (b), a Fourier Shell Correlation (FSC) plot (c) and a slice through the local resolution map (d). Scale bars are 50 and $10 \mathrm{~nm}$ respectively. 


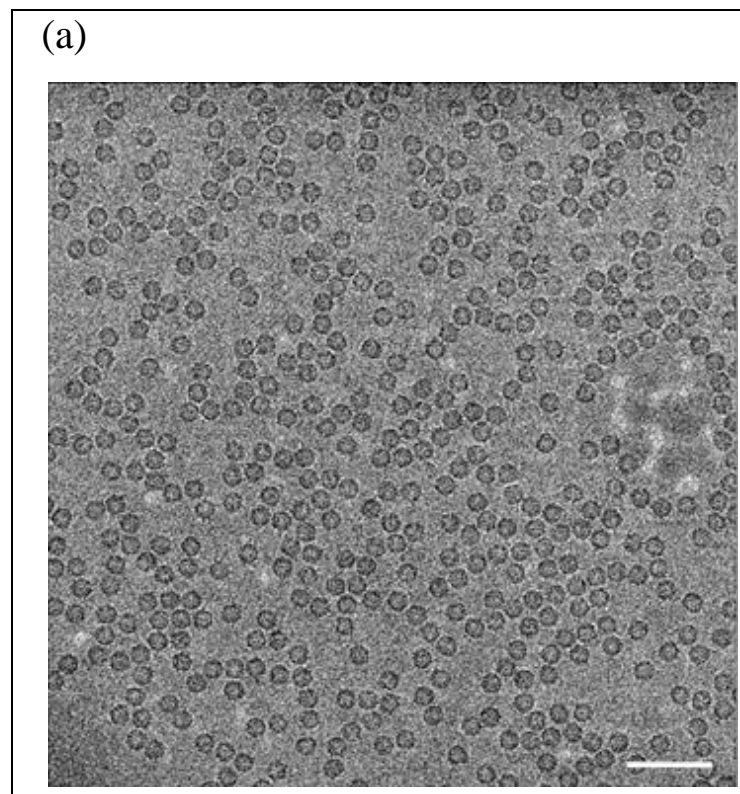

(c)

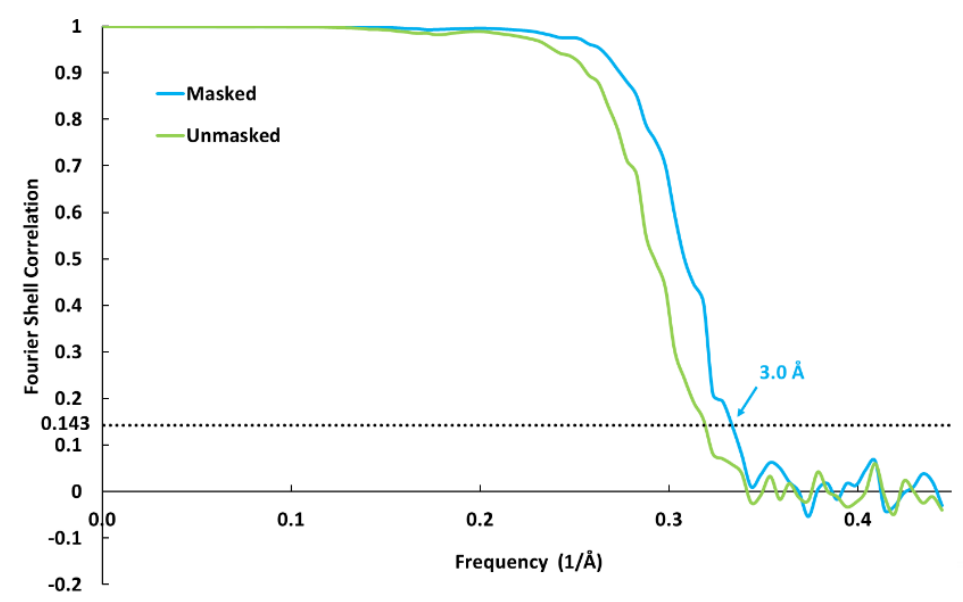

(b)

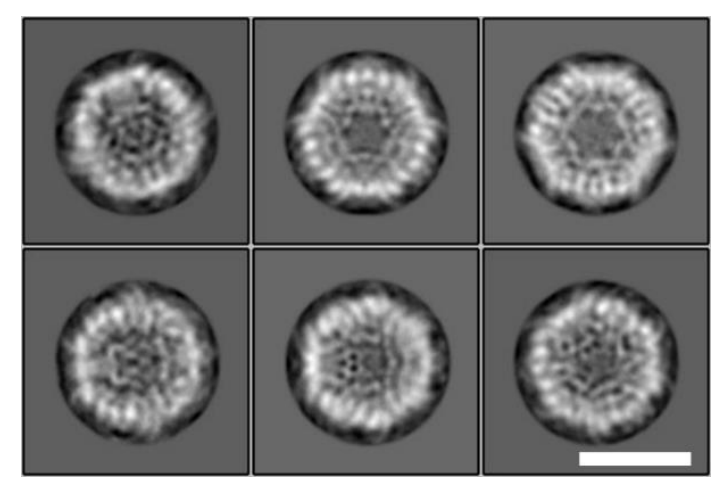

(d)

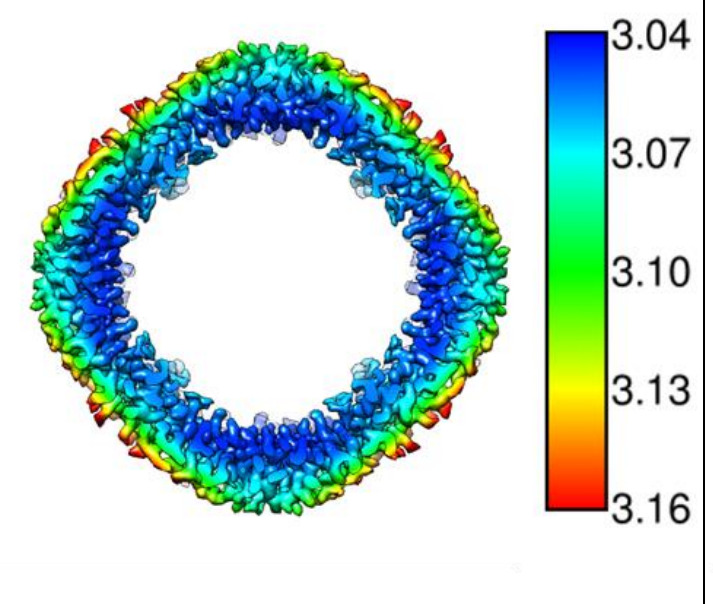

Figure S5: Summary of image processing results of the Acetate Washed sample. A representative micrograph (a), typical "good" class averages (b), an FSC plot (c) and a slice through the local resolution map (d). Scale bars are 50 and $10 \mathrm{~nm}$ respectively. 


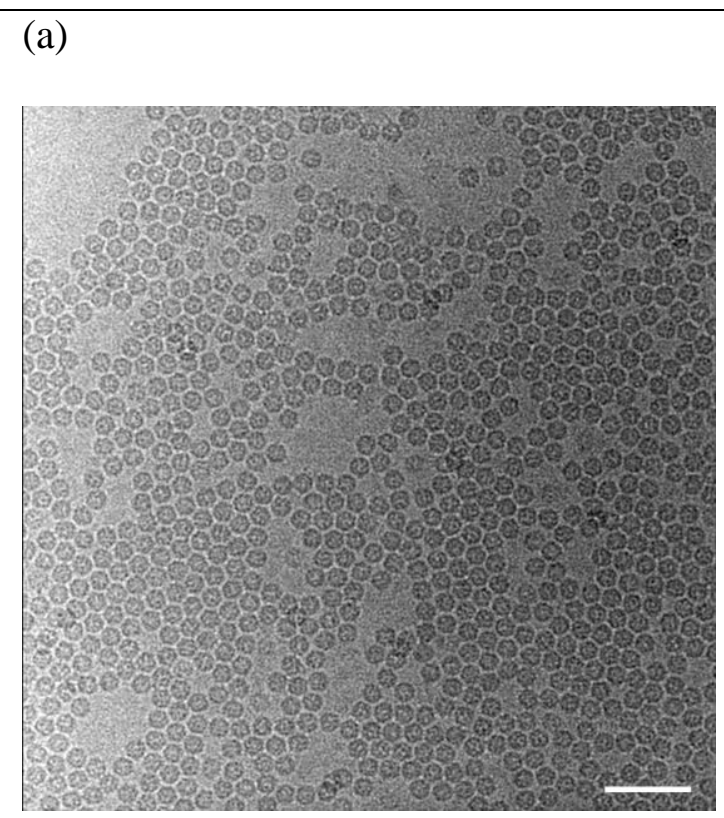

(c)

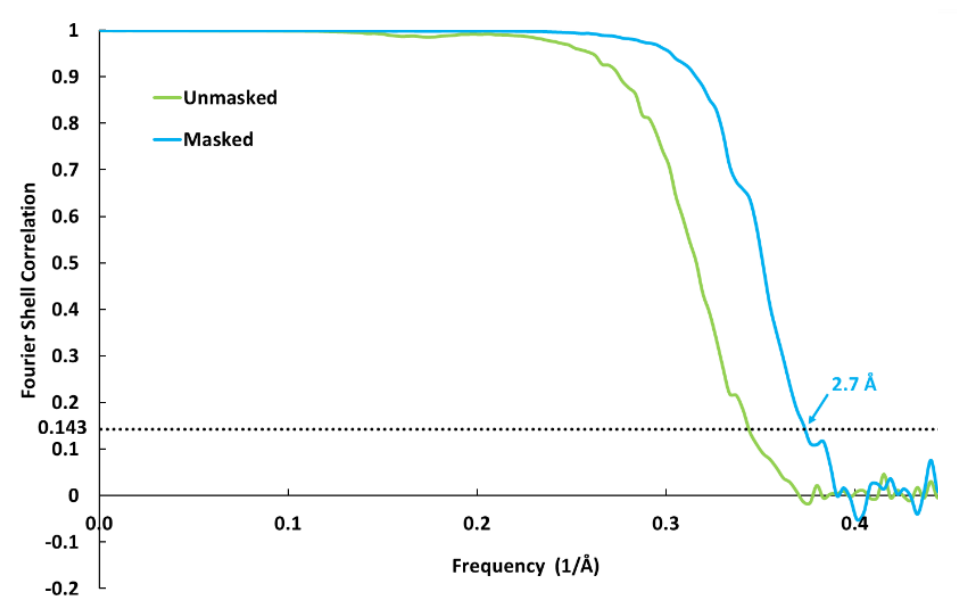

(b)

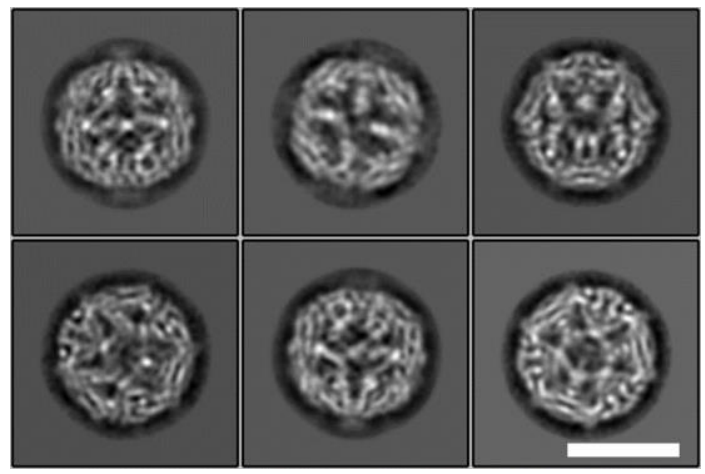

(d)

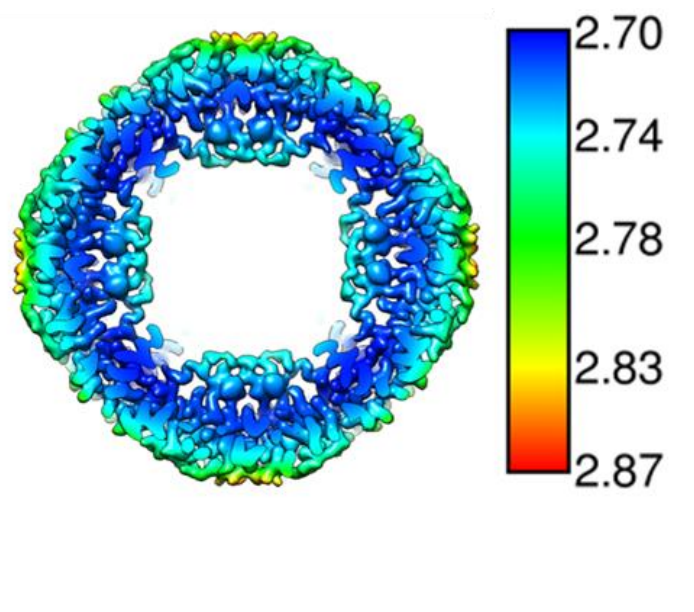

Figure S6: Summary of image processing results of the Refilled sample. A representative micrograph (a), typical "good" class averages (b), an FSC plot (c) and a slice through the local resolution map (d). Scale bars are 50 and 10 $\mathrm{nm}$ respectively. 


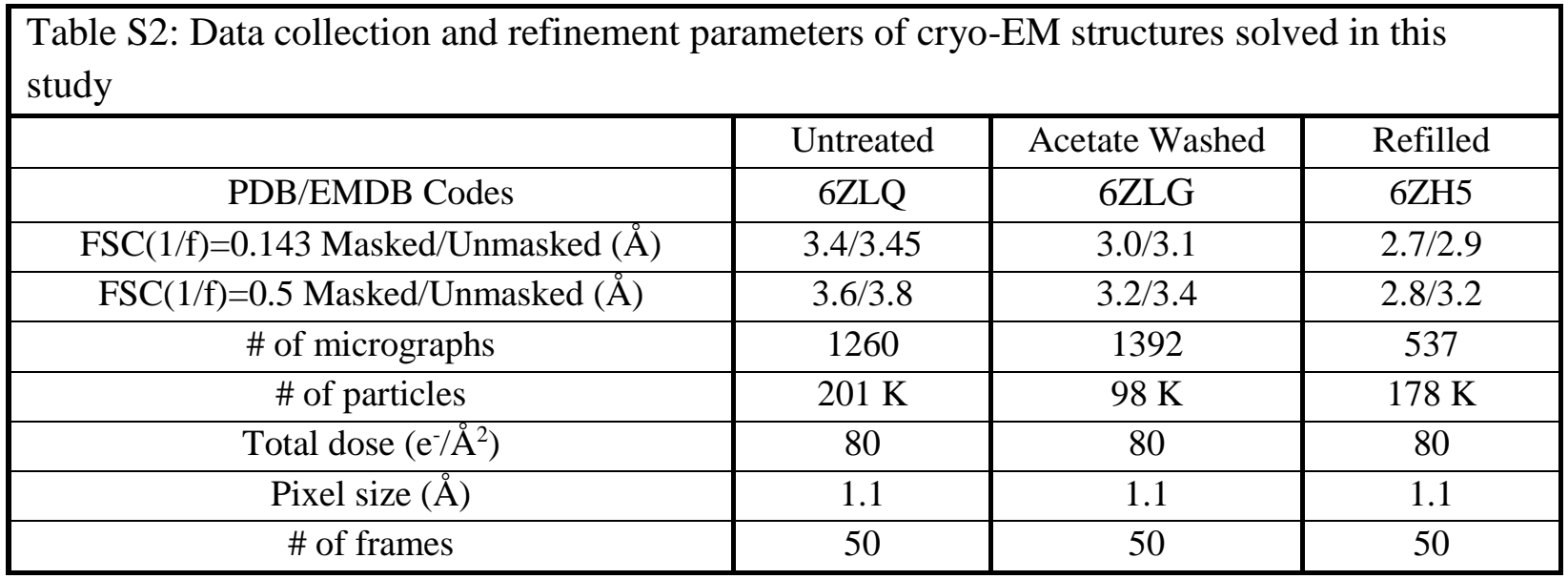

\section{Radiation assisted single particle analysis protocol}

\section{Purpose}

This protocol provides step by step guidance for generation of high dose 3D EM maps after the standard analysis of a dose fractionated cryo-EM dataset using Relion3.0.

\section{Prerequisites}

- A dose fractionated dataset of sufficient quality.

- A suitable GPU computer, as recommended in the Relion wiki.

- Relion 3 should be installed correctly and the path to its programs should be appended in \$PATH.

\section{Step 1 -determination of the alignment parameters for the dose fractionated particles}

Follow standard protocols for movie alignment, CTF determination, particle picking, initial model building, refinement and polish. Relion3.0 users can follow the detailed tutorial [7]. At the end of Step 1, the Relion project will have an output of the 3D map that will be analyzed by the current protocol.

Make sure the following outputs of step 1 were generated by Relion: 
a. The 3D map that will be analyzed by the current protocol designated here as: /Refine3D/<BestStructure $>$

b. In the Polish directory used for generating <BestStructure $>$ designated here as: /Polish $/\langle$ BestPolishJob $\rangle$ must be located.

c. The mask used for post processing 〈BestStructure > designated here as: 〈Mask.mrc〉

\section{Step 2 - Construction of "late frames" particle stack}

Construction of late frame particle stacks can be performed in various ways.

A convenient way is to select the frames of interest using the Relion Polish GUI menu as described in the Relion tutorial bayesian polishing section. Note the job number and name, this job will be designated here as <HighDosePolish $\rangle$.

In this paper we used frames $26-50$ which are equivalent to a total dose of $42-80 \mathrm{e}^{-/} \mathrm{A}^{2}$. Read the content of the path to the Micrographs directory which is one the outputs of the $<$ HighDosePolish> job

i.e.

$>$ ls -1 *.mrcs /Polish/<HighDosePolish>/Micrographs

The *.mrcs stacks of the high dose particles are stored in this subdirectory. Each *.mrcs file stores the particles of a single micrograph.

\section{Step 3 - Rerouting of the refinement parameters}

Generate an additional copy of the run_data.star file which contains the alignment parameters of the particles used for reconstructing the 3D map.

This can be done by the following commands issued from the Relion project root: 
$>c p /$ Refine $3 D /<$ BestStructure $>/ r u n \_d a t a . s t a r$

/Refine3D/<BestStructure>/run_data_HighDose.star

Each record in the file run_data_HighDose.star contains the information on a single particle.

One of the fields in each record is the path to the particle stack (i.e. a path to a *.mrcs file) and its number in the stack. This field will point to the <BestPolishJob $>$

Use a text editor or a command line to replace the path to the $\langle$ BestPolishJob $\rangle$ with the path to the <HighDosePolish > for all records.

After the editing is done,run_data_HighDose.star will contain the alignment parameters from the last BestStructure refinement job (step 1), however its content will point to the high dose particles from step 2 i.e. HighDosePolish.

\section{Step 4 - Reconstruction of the high dose 3D map}

Issue the following commands from the Relion project root:

> mkdir HighDoseMap

$>$ relion_reconstrcut --i /Refine $3 D /\langle$ BestStructure $\rangle /$ run_data_HighDose.star --ctf --sym $\langle$ SYM $\rangle$

--subset 1 --o HighDoseMap/run_half1_class001_unfil.mrc

$>$ relion_reconstrcut --i /Refine3D/<BestStructure $\rangle /$ run_data_HighDose.star --ctf --sym $\langle$ SYM $\rangle$ --subset 2 --o HighDoseMap/run_half2_class001_unfil.mrc

Where $\langle$ SYM $>$ is the symmetry of the reconstructed map e.g. C3. 
This will create the subdirectory HighDoseMap on the Relion project root directory and will output the two halves of the high dose 3D maps into the new HighDoseMap directory (i.e. run_half1_class001_unfil.mrc and run_half2_class001_unfil.mrc), generated based on the reconstruction parameters in run_data_HighDose.star.

Issue the following commands from the Relion project root:

$>$ relion_postprocess --mask<Mask.mrc> --i HighDoseMap/run_half1_class001_unfil.mrc--o HighDoseMap --angpix <PixelSize > --mtf <MTF_curve.star>

Where:

<mask.mrc> is the full path to the mask used during the 3D refinement in step 1

$<$ PixelSize $>$ is the pixel size at the sample plane in Angstroms

$<\mathrm{MTF}$ _curve.star $>$ is the full path to the MTF curve of the detector used for data collection

The high dose map: /HighDoseMap/postprocess.mrc will be created 


\section{References}

[1] M. Truman-Rosentsvit, D. Berenbaum, L. Spektor, L.A. Cohen, S. Belizowsky-Moshe, L. Lifshitz, J. Ma, W. Li, E. Kesselman, I. Abutbul-Ionita, D. Danino, L. Gutierrez, H. Li, K. Li, H. Lou, M. Regoni, M. Poli, F. Glaser, T.A. Rouault, E.G. Meyron-Holtz, Ferritin is secreted via 2 distinct nonclassical vesicular pathways, Blood. 131 (2018) 342-352. doi:10.1182/blood-2017-02-768580.

[2] M. Asperti, T. Stuemler, M. Poli, M. Gryzik, L. Lifshitz, E.G. Meyron-Holtz, I. Vlodavsky, P. Arosio, Heparanase Overexpression Reduces Hepcidin Expression, Affects Iron Homeostasis and Alters the Response to Inflammation, PLoS One. 11 (2016) e0164183. doi:10.1371/journal.pone.0164183.

[3] M. Radoul, L. Lewin, B. Cohen, R. Oren, S. Popov, G. Davidov, M.H. Vandsburger, A. Harmelin, R. Bitton, J.M. Greneche, M. Neeman, R. Zarivach, Genetic manipulation of iron biomineralization enhances MR relaxivity in a ferritin-M6A chimeric complex, Sci. Rep. 6 (2016). doi:10.1038/srep26550.

[4] P. Emsley, B. Lohkamp, W.G. Scott, K. Cowtan, Features and development of Coot, Acta Crystallogr. Sect. D Biol. Crystallogr. 66 (2010) 486-501. doi:10.1107/S0907444910007493.

[5] A.A. Vagin, R.A. Steiner, A.A. Lebedev, L. Potterton, S. McNicholas, F. Long, G.N. Murshudov, REFMAC5 dictionary: Organization of prior chemical knowledge and guidelines for its use, Acta Crystallogr. Sect. D Biol. Crystallogr. 60 (2004) 2184-2195. doi:10.1107/S0907444904023510.

[6] E.F. Pettersen, T.D. Goddard, C.C. Huang, G.S. Couch, D.M. Greenblatt, E.C. Meng, T.E. 
Ferrin, UCSF Chimera - A visualization system for exploratory research and analysis, J. Comput. Chem. 25 (2004) 1605-1612. doi:10.1002/jcc.20084.

[7] Relion tutorial, Https://Www3.Mrc-Lmb.Cam.Ac.Uk/Relion/Index.Php?Title=Main_Page. (n.d.). 